\title{
Motion of a Solid Large Spherical Aerosol Particle in a Single- Component Gas under the Influence of Carrier Medium
}

\author{
E.R. Schukin ${ }^{1}$, N.V. Malai $^{2}$, Z.L. Shulimanova ${ }^{3}$ and A.B. Nadykto ${ }^{4}$ \\ ${ }^{1}$ Joint Institute High Temperatures, Russian Academy of Sciences, RU- 125412, Moscow, Russia \\ ${ }^{2}$ Belgorod National Research University, RU-308015, Belgorod, Russia \\ ${ }^{3}$ Russian University of Transport (MIIT), RU- 127994, Moscow, Russia \\ ${ }^{4}$ Moscow State Technological University "STANKIN", RU-127055, Moscow, Russia
}

\begin{abstract}
In the article, a motion of a large solid spherical aerosol particle in a single-component gas has been investigated. Mathematical modeling has been performed considering inertia, particle radius and resistance of the carrier gaseous medium to its motion. The obtained formulas allow determining the time of the particle transition into the final states, and also the distances travelled by the particle. The numerical evaluations solved with the use of these formulas demonstrate that for large initial Reynolds numbers the particle stopping distance can be significant.
\end{abstract}

\section{Introduction}

The composition of natural, atmospheric, and industrial aerosols surrounding us [1-7] may include various types of solid aerosol particles [2-6]. These particles can have an arbitrary surface shape, in particular, close to spherical, cylindrical, ellipsoidal, needle, lamellar and other forms [9]. They also have properties that are very important for practical applications, including physicochemical ones. Therefore, aerosol particles are increasingly used in practice [3-6]. They are used, for example, in various fields of technology, including military and space, in medicine[26], in particular in the treatment of wounds, with lesions of the skin and inhalation. Aerosol particles protect agricultural and forest crops from pests, protect and treat animals and birds from parasites and diseases. They are used in everyday life, in military affairs with blackout, etc. Aerosol particles play an important role in the formation of precipitation [27]. Aerosol particles are also widely used in industry, in particular, as catalysts and components of high-energy fuels. Aerosol particles play a well-known role in biology - plant pollen, bacterial and mold spores, and light seeds are transported in nature in the form of aerosols. In connection with this, a comprehensive study of the various properties of aerosol particles, including the laws of their ordered motion in gaseous media, is of great scientific and practical interest [2-6]. In practical applications, they often deal with the ordered movement of aerosol particles that occurs under the influence of fairly well-studied external forces [6,9]. Such forces include, for example, and of molecular nature, thermophoretic [5,6,8-20,24] and photophoretic $[15,19,21-23]$ forces. Thermophoretic force moves particles to areas with lower temperatures. Its appearance is due to the transfer by particles of molecules of an inhomogeneous temperature gas of an uncompensated impulse. The thermophoretic movement of aerosol particles occurs during nonisothermal industrial and natural processes $[3,5,6,24]$. Such movement of aerosol particles can occur when they are deposited on heat transfer surfaces with lower temperatures, for example, in thermal precipitators, heat and mass exchanger channels $[3,6,9]$, cloud and fog clear zones [4,27]. The thermophoretic movement can, in particular, be used for fine purification of gases, separation of particles by size, obtaining new materials with preset properties, in medicine.

The appearance of the photophoretic force is due to the interaction of the molecules surrounding the gas particles with the particle surface inhomogeneously heated by electromagnetic radiation. This force has a major effect on the movement of particles, called photophoretic. The latter is widely used in technical, medical, environmental and biological applications. The photophoretic force has a significant effect, in particular, on the movement of solid black large and moderately large aerosol particles.

Of great importance is the study of the laws and free motion of particles, which occurs at large initial velocities and Reynolds numbers, since these particles can travel long distances before stopping. Large initial velocities $\vec{V}_{i}$ and Reynolds numbers $R e_{i}$ can have aerosol particles of different nature, in particular, shotguns, particles that are formed during dispersion (grinding, spraying) of solids, particles flying from the surface of grinding wheels, particles emitted into the atmosphere by ventilation systems [6,9]. At high 
speeds, particles formed during the high-temperature combustion of fuel in the furnaces can move, internal combustion engines as well particles in the exhaust gases of diesel engines of ships, engines of jet and turbojet aircraft [2-4.9], etc.

It should be noted that aerosol particles moving in gaseous media are affected by the medium resistance which slows down their motion [1,2]. In this process, particles transition from states with the initial Reynolds number $R e_{i}$ to states with a smaller Reynolds number $R e_{f}$. Therefore, when assessing the motion of aerosol particles, the gaseous medium resistance influencing their motion must also be considered.

The resistance force impact ceases when the particle stops. In this case $R e_{f}=0$. The distance traveled by the particle to a complete stop is called the stopping distance [1,2]. The length of this distance can greatly depend on the initial Reynolds number $R e_{i}$ and particle sizes. Thus, knowing the formulas allowing, at known aerosol particle sizes and initial Reynolds numbers $R e_{i}$, directly assessing the time the particles take to transition from states with a known initial number $R e_{i}$ to states with smaller Reynolds numbers $R e_{f}$, as well as the distances traveled by particles, in particular, stopping distances, is of significant interest when assessing aerosol particle motion for practical applications considering the impact of the gaseous medium resistance.

The formulas provided below allow, at the initial Reynolds numbers $0<R e_{i} \leq 1000$, assessing the motion of large solid spherical aerosol particles with consideration of how their motion is impacted not only by the gaseous medium resistance, but also by their radius.

\section{Problem Statement}

Free one-dimensional motion of a large solid spherical aerosol particle (with the Knudsen number $K n=\lambda / R<$ $0.01[5,6]$, where $\lambda$ is the average free path length of the gas molecules, $R$ is the particle radius) with the diameter $d^{*}$, initial Reynolds number $R e_{i}$, and velocity $\vec{V}_{i}=V_{i x} \vec{n}_{x}$ occurs in a single-component gas in a horizontal direction along the $\mathrm{Ox}$ axis. The particle moving in the gas is affected by the following gas resistance force decelerating its motion (1) $[5,6]$ :

$$
\vec{F}_{D}=F_{D} \vec{n}_{x}
$$

Expression (1) for the resistance force includes the scalar coefficient $F_{D}=\left(-\pi \mu \nu \beta_{D} \mathrm{Re}^{2}\right) / 8$, where $v=\mu / \rho_{m}$ and $\mu$ are the kinematic and dynamic viscosity coefficients of the gaseous medium, $\rho_{m}$ is the gas density, $R e=\left(V_{x} d^{*} / v\right)$ is the particle Reynolds number, $V_{x}$ is the projection of the particle velocity vector on the Ox axis, $\vec{n}_{x}$ is a unit vector in the direction of the $\mathrm{Ox}$ axis, $\beta_{D}$ is the coefficient of the gas medium resistance to the particle motion that depends on the Reynolds number [1,2].

Coefficient $\beta_{D}$, which is a part of the formula for the resistance force (1), can have a significant influence on its value. The experimental values of the resistance coefficient $\beta_{D}$ [4] for large spherical particles in the air depending on the Reynolds number are presented in Table 1. Derivation of analytical expressions for the coefficient $\beta_{D}$ is a complicated mathematical problem. Analytical expressions (2) and (3) for the coefficient $\beta_{D}$ of large solid spherical particles obtained in Stokes' and Oseen's approximations, respectively, have the following form $[2,6]$ :

$$
\begin{gathered}
\beta_{D}^{(S)}=(24 / \mathrm{Re}) \\
\beta_{D}^{(0)}=\beta_{D}^{(S)}[1+(3 \mathrm{Re} / 16)] .
\end{gathered}
$$

A comparison of the experimental values of the resistance coefficient with the values from theoretical formulas (2) and (3) demonstrates that formula (2) at $\operatorname{Re}>0.3$ allows obtaining only underestimated values of the resistance coefficient, and formula (3) at $\mathrm{Re}$ $<1000$ allows finding only overestimated values of the resistance coefficient. The following empirical formula obtained by the authors on the basis of the experimental data provided in [4] gives a relatively good description of the dependence of the experimental values $\beta_{D}^{(\ni)}$ on the Reynolds number at $\operatorname{Re}<1000$ :

$$
\beta_{D}=\beta_{D}^{(S)} \sqrt{1+A \operatorname{Re}+B \operatorname{Re}^{2}}
$$

where $\mathrm{A}=0.1865$ and $\mathrm{B}=1.52 \times 10^{-4}$ are empirical constants.

Table 1. The $\beta_{D}$ values obtained experimentally and using formulas (2)-(4) depending on the Reynolds number.

\begin{tabular}{|c|c|c|c|c|}
\hline \multirow{2}{*}{$\operatorname{Re}$} & Ex. & \multicolumn{3}{|c|}{ Formulas } \\
\cline { 2 - 5 } & $\beta_{D}^{(\text {Э) }}$ & $\beta_{D}^{(S)}$ & $\beta_{D}^{(0)}$ & $(4)$ \\
\hline 0.1 & 240 & 240 & 244.6 & 242.12 \\
\hline 0.2 & 120 & 120 & 124.4 & 122.23 \\
\hline 0.3 & 80 & 80 & 84.7 & 82.22 \\
\hline 0.5 & 49.5 & 48.2 & 52.3 & 50.19 \\
\hline 0.7 & 36.5 & 34.3 & 38.75 & 36.17 \\
\hline 1.0 & 26.5 & 24.3 & 28.51 & 25.91 \\
\hline 2 & 14.6 & 12.21 & 16.52 & 13.93 \\
\hline 3 & 10.4 & 8.12 & 12.51 & 9.91 \\
\hline 5 & 6.9 & 4.83 & 9.32 & 6.72 \\
\hline 7 & 5.3 & 3.42 & 7.91 & 5.19 \\
\hline 10 & 4.1 & 2.4 & 6.9 & 4.06 \\
\hline 20 & 2.55 & 1.2 & 5.7 & 2.62 \\
\hline 30 & 2.00 & 0.8 & 5.3 & 2.07 \\
\hline 50 & 1.50 & 0.48 & 4.98 & 1.58 \\
\hline 70 & 1.27 & 0.34 & 4.8 & 1.31 \\
\hline 100 & 1.07 & 0.24 & 4.74 & 1.11 \\
\hline 200 & 0.77 & 0.12 & 4.62 & 0.82 \\
\hline 300 & 0.65 & 0.08 & 4.58 & 0.66 \\
\hline 500 & 0.55 & 0.05 & 4.55 & 0.54 \\
\hline 1000 & 0.46 & 0.024 & 4.52 & 0.43 \\
\hline & & & & \\
\hline
\end{tabular}


It should be noted that formula (4) can be used for analytical solution of aerosol mechanics problems for cases with big and small Reynolds numbers. This will be demonstrated below using an example of solving a problem of free one-dimensional motion in a singlecomponent gas of a large solid spherical aerosol particle with its Reynolds numbers $\mathrm{Re}<1000$.

\subsection{Time of the article transition into states with new Reynolds numbers}

Free motion of a large solid spherical aerosol particle in a gas is decelerated by the gaseous medium resistance force (1) [1,2]. The written equation for the motion of the considered particle, taking into account expression (1) for the resistance force, has the following form:

$$
m \frac{d V_{x}}{d t}=-(\pi / 8) \beta_{D} \mu \nu \operatorname{Re}^{2}
$$

In this equation $\mathrm{t}$ is the time, $m=\frac{\pi}{6} d^{* 3} \rho_{p}$ is the particle mass, $\rho_{p}$ is the particle substance density, $d^{*}$ is the particle diameter. Equation (5) takes the form convenient for integration after entering the Reynolds number into its left side, consideration of expressions for $\beta_{D}$ (4), particle mass $m$ and $v=\mu / \rho_{m}$. Thereby we obtain:

$$
d t=-\left(\xi / \operatorname{Re} \sqrt{1+A \operatorname{Re}+B \operatorname{Re}^{2}}\right) d R e .(6)
$$

Here $\xi=d^{* 2} \rho_{p} / 18 \mu$ is the relaxation time of the spherical aerosol particle at $\operatorname{Re}<<1$ [2]. After integration of the right side of equation (6) from the initial value $R e_{i}$ to $R e_{f}$, we obtain expression (7) connecting the values of the ratio $\left[\Delta\left(\operatorname{Re}_{f}\right) / \Delta\left(\operatorname{Re}_{i}\right)\right]$ with transition time $t$ of the particle from a state with $R e_{i}$ to a state with $R e_{f}$ :

$$
\exp (t / \xi)=\left\lfloor\Delta\left(\operatorname{Re}_{f}\right) / \Delta\left(\operatorname{Re}_{i}\right)\right\rfloor,
$$

$\Delta(\mathrm{Re})=\left\lfloor 2\left(\sqrt{1+A \operatorname{Re}+B \operatorname{Re}^{2}}+1\right)+A \operatorname{Re}\right\rfloor / \operatorname{Re}$

Formula (7) with consideration of formula (8) allows, at a known radius, density, particle numbers $R e_{i}$ and $R e_{f}$, directly obtaining the time of its transition to a state with the smaller number $R e_{f}$. The data in Table 2 on the dependence of the $R e_{f}$ variables of steel particles with $d^{*}=0.4 ; 0.2 ; 0.1 \mathrm{~cm}$, airborne at a temperature $\mathrm{t}^{0}=20^{\circ} \mathrm{C}$ and pressure $\mathrm{p}=1 \mathrm{~atm}$ on time $t$ were obtained using these formulas.

These data demonstrate that the transition time $t$ can be greatly dependent on the particle diameter.
Table 2. Dependence of the $R e_{f}$ numbers at $R e_{i}=1000$ on time $t$.

\begin{tabular}{|c|c|c|c|}
\hline$R e_{f}$ & $\begin{array}{c}d^{*}=0.4, \\
\mathrm{~cm}\end{array}$ & $\begin{array}{c}d^{*}=0.2, \\
\mathrm{~cm}\end{array}$ & $\begin{array}{c}d^{*}=0.1, \\
\mathrm{~cm}\end{array}$ \\
\cline { 2 - 4 } & $\mathrm{t}, \mathrm{c}$ & $\mathrm{t}, \mathrm{c}$ & $\mathrm{t}, \mathrm{c}$ \\
\hline 800 & 6.84 & 1.71 & 0.4275 \\
\hline 200 & 59.28 & 14.82 & 3.705 \\
\hline 25 & 268.7 & 67.18 & 16.79 \\
\hline 5 & 617.5 & 154.4 & 38.59 \\
\hline 1 & 1126 & 281.5 & 70.38 \\
\hline 0.1 & 1972 & 493 & 123.3 \\
\hline
\end{tabular}

Taking the logarithm (7) allows transitioning to the following simpler expression for $t$ : $t=\xi \ln \left[\Delta\left(\operatorname{Re}_{f}\right) / \Delta\left(\operatorname{Re}_{i}\right)\right]$.

\subsection{The distance traveled by the particle during its transition to a state with new Reynolds numbers}

During the transition of the particle from the initial state with $R e_{i}$ to the state with $R e_{f}$, the particle travels a certain distance with the length $S$.

The differential $d S$ of the distance $S$, covered during the time $d t$, equals:

$$
d S=V_{x} d t .
$$

Considering the above-mentioned expression for $R e$ (see the Problem Statement section) connecting the Reynolds number with the projection $V_{x}$, differential $d t$ (6) and resistance coefficient $\beta_{D}$ (4), formula (9) for $d S$ was transformed into the following simpler formula (10), which connects the values of the differentials $d S$ and $d R e$ :

$$
d S=-d^{*}\left(\widehat{S} \sqrt{1+A \operatorname{Re}+B \operatorname{Re}^{2}}\right) d \operatorname{Re},
$$

where $\hat{S}=\left(\rho_{p} / 18 \rho_{m} \sqrt{B}\right)$. During integration of (10) from $R e_{i}$ to $R e_{f}$, the following expression (11), which allows at known values $d^{*}, \widehat{S}, R e_{i}$ and $R e_{f}$ determining the traveled path length $S$, was obtained:

$$
\exp \left(S / d^{*} \cdot \widehat{S}\right)=\left[L\left(\operatorname{Re}_{i}\right) / L\left(\operatorname{Re}_{f}\right)\right],
$$

where $\widehat{S}=\left(\rho_{p} / 18 \rho_{m} \sqrt{B}\right)$,

$$
L(\mathrm{Re})=\left\lfloor 2 \sqrt{B\left(1+A \operatorname{Re}+B \operatorname{Re}^{2}\right)}+2 B \operatorname{Re}+A\right\rfloor .
$$

For instance, the length $S$ traveled by particles until they come to a stop (stopping distance) can be found using formula (11) by substituting $R e_{f}=0$ into it. In this case the formula for $\mathrm{S}$ (11) transforms into the following simpler formula (12):

$$
\exp \left(S / d^{*} \cdot \widehat{S}\right)=\left\lfloor L\left(\operatorname{Re}_{i}\right) /(2 \sqrt{B}+A)\right\rfloor,
$$


The conducted analysis of the formula for the stopping distance $\mathrm{S}$ (12) showed that at initial numbers $\mathrm{Re}_{i}<2$ the dependence of the stopping distance on the number $\mathrm{Re}_{i}$ is close to linear. And at big initial Reynolds numbers $\mathrm{Re}_{i}$, it is the dependence of the stopping distance $\mathrm{S}$ on $\ln \mathrm{Re}_{i}$ that is close to linear. It has also been established that the stopping distances $\mathrm{S}$ dependent on the initial numbers $\mathrm{Re}_{i}$ and particle diameters $d^{*}$, can be significant.

Table 3. The dependence of the stopping distances $\mathrm{S}$ on the $R e_{i}$ numbers and particle diameters $d^{*}$.

\begin{tabular}{|l|c|c|c|c|}
\hline \multirow{2}{*}{$R e$} & $\begin{array}{l}d^{*}= \\
0.015 \mathrm{~m}\end{array}$ & $\begin{array}{l}d^{*}= \\
0.01 \mathrm{~m}\end{array}$ & $\begin{array}{l}d^{*}= \\
0.005 \mathrm{~m}\end{array}$ & $\begin{array}{l}d^{*}= \\
0.002 \mathrm{~m}\end{array}$ \\
\cline { 2 - 5 } & $\mathrm{S}, \mathrm{m}$ & $\mathrm{S}, \mathrm{m}$ & $\mathrm{S}, \mathrm{m}$ & $\mathrm{S}, \mathrm{m}$ \\
\hline 1000 & 252 & 168 & 84 & 33.6 \\
\hline 800 & 223 & 148 & 74 & 29.7 \\
\hline 600 & 198.8 & 132.5 & 66.3 & 26.5 \\
\hline 400 & 162.5 & 108.3 & 54.2 & 21.7 \\
\hline 200 & 112.3 & 74.9 & 37.4 & 14.9 \\
\hline 100 & 75.3 & 50.2 & 25.1 & 10.04 \\
\hline 50 & 48.9 & 32.6 & 16.3 & 6.52 \\
\hline 10 & 15.4 & 10.3 & 5.13 & 2.05 \\
\hline 5 & 8.67 & 5.78 & 2.89 & 1.16 \\
\hline 1 & 1.98 & 1.32 & 0.66 & 0.265 \\
\hline
\end{tabular}

The stopping distances $\mathrm{S}$ obtained at the air temperature $t^{0}=20^{\circ} \mathrm{C}$ and particle density $\rho_{P}=32 / \mathrm{CM}^{3}$ are presented in Table 3. The evaluations were performed using formula (12) for the length $\mathrm{S}$, with consideration of the variable $\mathrm{L}\left(\mathrm{Re}_{\mathrm{i}}\right)$.

It should also be noted that formulas (11) and (12) for the length $\mathrm{S}$ after taking the logarithm acquire the following simplest form (13) and (14), respectively:

$$
\begin{aligned}
& S=d^{*} \cdot \widehat{S} \ln \left[L\left(\operatorname{Re}_{i}\right) / L\left(\operatorname{Re}_{f}\right)\right], \\
& S=d^{*} \cdot \widehat{S} \ln \left[L\left(\operatorname{Re}_{i}\right) /(2 \sqrt{B}+A)\right]
\end{aligned}
$$

\section{Conclusion}

In the article, mathematical modeling of the process of free motion in a single-component gas of a large solid spherical aerosol particle is carried out taking into account inertia, particle radius, resistance force of the carrier gaseous medium to its movement. The formulas found in this process of solving the equations of gas dynamics allow for the given radius, density, initial and final Reynolds numbers of the particles to find the time of its transition to final states, as well as the distances covered by the particle, including the braking distance. Using these formulas, data were found $\mathrm{Re}_{i}=1000$ (see Table 2) as a function of time $t$ of the final Reynolds numbers of steel particles in air. These data, in particular, show that the time $\mathrm{t}$ can strongly depend on the particle diameter.

Assessment of the distance traveled and the braking distances of aerosol particles showed that they can be significant at large initial speeds and Reynolds numbers and can reach several hundred meters at large particle diameters (see Table 3). This circumstance must be taken into account using aerosol particles in practical applications

We thank Russian Science Foundation (grant №18-7100137) and the Russian Ministry of Science and Education (grants 1.6198.2017/6.7 and 1.7706.2017/8.9) for support. This work was carried out using equipment provided by the Center of Collective Use of MSUT "STANKIN".

\section{References}

1. R.H. Perry, C. N. Chilton, Chemical engineers handbook. 5-th Ed. (New York.: McGraw-Hill. 1973)

2. P.C. Reist, Aerosols Introduction to the Theory (Moscow, Energetics and Energy, 1987)

3. I.V. Petrynov-Soкolov, Aerosols (Moscow, Nauka, 1989)

4. V.M. Polonsky, Air Pollution Control (Moscow, ASV Publishing House, 2006)

5. N.A. Fuks, Mechanics of Aerosols. (Moscow, Eksmo, 2009)

6. V.N. Piskunov, Dynamics of Aerosols. (Moscow, Fismatlit Publishers, 2010)

7. I.S. Ivlev, Optics of the atmosphere and ocean, 8. 1039 - 1057 (1996)

8. E.R. Shchukin, Proceedings of the International scientific-practical conference "Actual problems of the development of science and education", May 5, Moscow 1, 117-123 (2014)

9. A.I. Pirumov Dedusting of the air. (Moscow. Buildingpublish, 1981)

10. E.R. Shchukin, N.V. Malay, Z.L. Shulimanova, Vestnik TSU 19(3), (2014)

11. E.R. Shchukin, International scientific-practical conference "Actual problems of science and education. May 5. Moscow. 1, 123-126 (2014)

12. E.R. Shchukin, N.V. Malay, Z.L. Shulimanova Proceedings of the International Scientific Conference "Ecology of Water Basins and Water Transport" MGAVT, 98-101 (2014)

13. N.V. Malay, E.V. Kalyuzhnaya, D.A. Morel, E.R. Shchukin, Bulletin of the Tambov University. A series of natural and technical sciences. Tambov, 20(1), 92-97 (2015)

14. E.R. Shchukin, N.V. Malay, Z.L Shulimanova Bulletin of the Adygea State University, 2(161). 23-30 (2015)

15. N.V. Malay, N.N. Mironova, E.R. Shchukin, J. of Phusics and Appl., 2, 251-256 (2014) 
16. E.R. Shchukin, N.V. Malay and Z.L. Shulimanova, Nova Science Publishers, Inc., Books: Mathematics and Statistics / Series: Theoretical and Applied Mathematics // Nonlineary: problems, Solutions and Applications. 1, Chapter 20 (2017) ISBN: 978-1-53612-032-5 (Binding: Hardcover; Status: AV; New York. Pp. 447 - 455).

17. E.R. Shchukin, L.A.Uvarova, Z.L. Shulimanova, N.V.Malay, Nova Science Publishers, Inc., Books: Mathematics and Statistics / Series: Theoretical and Applied Mathematics // Nonlineary: problems, Solutions and Applications. 1. Chapter 21 (2017) ISBN: 978-1-53612-032-5 (Binding: Hardcover; Status: AV; New York. Pp. 457 - 467).

18. A.B. Poddoskin, N.V. Malay, E.R. Schukin, Scientific Bulletin of Belgorod State University. Series: Mathematics. Physics. 50(2), 152 -160 (2018)

19. N.V. Malay, E.R. Shchukin, Technical Physics, 64(4), 458- 464 (2019) ISSN 1063- 7842

20. N.V. Malay, E.R. Shchukin, Applied Mechanics and Technical Physics, 60(3), 136-145 (2019)

21. E.R. Shchukin, N.V. Malay, Z.L. Shulimanova, Zh. Bulletin of MSTU Stankin, 3, 92-96 (2016)
22. E.R. Shchukin, N.V. Malay, Z.L. Shulimanova, A.A. Stukalov Scientific reports of Belgorod State University. Mathematics Series. Physics, 43(13), 96-104 (2016)

23. N.V. Malay, E.R. Shchukin, Nova Science Publishers, Inc., Books: Mathematics and Statistics / Series: Theoretical and Applied Mathematics // Nonlineary: problems, Solutions and Applications. 1, Chapter 16 (2017) (Status: AV; New York. Pp. 317 - 334).

24. F. Zheng, Advances in Colloid and Interface Science, 97, 255-278 (2002)

25. T. Numazawa, K. Kamiya, Y. Hiratsuka, T. Satoh, H. Nozawa, T. Yanagitani, International Cryocooler Conference, 14, 367-372 (2007)

26. L. Zang, F.X. Gu, J.M. Chan, A.Z. Wang Clinical Pharmacology and Therapeutics, 83(5), 761-769 (2008)

27. L.S. Ivlev, Yu.A. Dovgalyuk Physics of atmospheric aerosol systems (Izd. SPbGU, St. Petersburg, 1999) 